\title{
Adenylate cyclase-associated protein 1 overexpressed in pancreatic cancers is involved in cancer cell motility
}

\author{
Ken Yamazaki ${ }^{1}$, Masaaki Takamura ${ }^{2}$, Yohei Masugi ${ }^{1}$,Taisuke Mori ${ }^{1}$, Wenlin Du ${ }^{1}$, Taizo Hibi ${ }^{3}$, Nobuyoshi Hiraoka ${ }^{4}$, \\ Tsutomu Ohta ${ }^{4}$, Misao Ohki ${ }^{4}$, Setsuo Hirohashi ${ }^{5}$ and Michiie Sakamoto ${ }^{1}$
}

Pancreatic cancer has the worst prognosis among cancers due to the difficulty of early diagnosis and its aggressive behavior. To characterize the aggressiveness of pancreatic cancers on gene expression, pancreatic cancer xenografts transplanted into severe combined immunodeficient mice served as a panel for gene-expression profiling. As a result of profiling, the adenylate cyclase-associated protein 1 (CAP1) gene was shown to be overexpressed in all of the xenografts. The expression of CAP1 protein in all 73 cases of pancreatic cancer was recognized by immunohistochemical analyses. The ratio of CAP1-positive tumor cells in clinical specimens was correlated with the presence of lymph node metastasis and neural invasion, and also with the poor prognosis of patients. Immunocytochemical analyses in pancreatic cancer cells demonstrated that CAP1 colocalized to the leading edge of lamellipodia with actin. Knockdown of CAP1 by RNA interference resulted in the reduction of lamellipodium formation, motility, and invasion of pancreatic cancer cells. This is the first report demonstrating the overexpression of CAP1 in pancreatic cancers and suggesting the involvement of CAP1 in the aggressive behavior of pancreatic cancer cells.

Laboratory Investigation (2009) 89, 425-432; doi:10.1038/labinvest.2009.5; published online 2 February 2009

KEYWORDS: CAP1; metastasis; microarray; pancreatic cancer; prognosis

Pancreatic cancer is a leading cause of cancer death worldwide. ${ }^{1,2}$ Only about $20 \%$ of pancreatic cancer can be surgically resected with curative intent at the time of diagnosis. ${ }^{3}$ Mainly due to the difficulty in early detection and frequent metastatic dissemination, the 5-year survival rate for pancreatic cancer patients remains below $5 \% .{ }^{3,4}$ Considering that vascular involvement, lymph node metastasis, and neural invasion have been proposed as prognostic factors, ${ }^{5-7}$ it seems that metastasis is responsible for the aggressive behavior of pancreatic cancer. Pancreatic cancer appears to acquire genetic aberrations with successive alteration of genes involved in the regulation of cell proliferation. The alterations include activating mutations of the KRAS gene ${ }^{8,9}$ that occur early in the stage of pancreatic carcinogenesis, and inactivations by the deletion and mutation of the CDKN2A, ${ }^{10}$ TP53, ${ }^{11}$ and SMAD4 genes. ${ }^{12}$ In addition to cell proliferation, cell motility is one of the factors associated with cancer metastasis; however, no alteration of such genes has been revealed in pancreatic cancer. Therefore, alteration of the expression level of genes by epigenetic or transcriptional manner may be a cue to elucidate the mechanism of cancer metastasis.

Recent developments in the technology of human genome research enabled us to obtain genome-wide gene-expression profiles, and vast numbers of marker molecule candidates for pancreatic cancer have been proposed. ${ }^{13-18}$ In this study, xenografts of clinical specimens orthotopically transplanted into severe combined immunodeficient (SCID) mice $^{19}$ were utilized as a panel for gene-expression profiling of pancreatic cancer. Among commonly overexpressed genes in pancreatic cancer xenografts, we further evaluated the adenylate cyclaseassociated protein 1 (CAP1) gene, which encodes an actin monomer-binding protein (reviewed in reference ${ }^{20}$ ). The reorganization of actin filament is essential for cell migration and is regulated by actin-binding proteins in some signaling pathways. $^{21,22}$ Therefore, we examined the role of overexpression of CAP1 in cell motility and the pathology of pancreatic cancer.

\footnotetext{
${ }^{1}$ Department of Pathology, School of Medicine, Keio University, Tokyo, Japan; ${ }^{2}$ Division of Gastroenterology and Hepatology, Niigata University Graduate School of Medical and Dental Sciences, Niigata, Japan; ${ }^{3}$ Department of Surgery, School of Medicine, Keio University, Tokyo, Japan; ${ }^{4}$ Cancer Genomics Division, National Cancer Center Research Institute, Tokyo, Japan and ${ }^{5}$ Pathology Division, National Cancer Center Research Institute, Tokyo, Japan

Correspondence: Professor M Sakamoto, MD, PhD, Department of Pathology, School of Medicine, Keio University, 35 Shinanomachi, Shinjuku-ku, Tokyo 160-8582, Japan.

E-mail: msakamot@sc.itc.keio.ac.jp
}

Received 19 September 2008; revised 5 December 2008; accepted 9 December 2008 


\section{MATERIALS AND METHODS Tissue Samples}

Pancreatic cancer and non-neoplastic pancreas tissues were obtained from patients who underwent surgical resection at Keio University Hospital and the National Cancer Center Hospital, Japan. All experiments using human samples were approved by the ethics committees of the National Cancer Center and of Keio University, School of Medicine. Cancer tissue fragments from 12 patients were orthotopically implanted into SCID mice as described previously. ${ }^{19}$

\section{Microarray Analyses}

Total RNA of each xenograft was extracted with Trizol (Invitrogen, Carlsbad, CA, USA) and then RNeasy (QIAGEN, Valencia, CA, USA). Biotinylated cRNA was prepared from $5 \mu \mathrm{g}$ of total RNA and then hybridized to GeneChip HGU133A (Affymetrix, Santa Clara, CA, USA) according to the manufacturer's protocol. Each profile was normalized as the mean signal of all probe sets at 1000 . Microarray data are accessible from our in-house database, Genome Medicine Database of Japan (GeMDBJ; https://gemdbj.nibio.go.jp/ $\mathrm{dgdb} /)$.

\section{Antibodies}

We used two antisera against human CAP1 protein. Both a rat polyclonal antibody (a kind gift from Dr Kenji Moriyama, The Tokyo Metropolitan Institute of Medical Science, Tokyo, Japan $)^{23}$ and a rabbit polyclonal antibody recognize the Cterminal domain of human CAP1. Each antibody raises similar results in Western blot and immunohistochemical analyses, and the specificity was further confirmed by antigen absorption tests as in a previous report ${ }^{24}$ for the rabbit polyclonal antibody (data not shown).

\section{Western Blot Analyses}

Lysates of pancreatic cancers, non-neoplastic pancreas tissues, and pancreatic cancer cells were loaded onto $10 \%$ polyacrylamide-SDS gels, separated by electrophoresis, and blotted onto PVDF membranes by the semidry transfer method. Anti-CAP1 and anti-actin (Sigma, St Louis, MO, USA) antibodies were hybridized to the membranes overnight at $4^{\circ} \mathrm{C}$. Horseradish peroxidase-conjugated secondary antibodies were probed to the membranes and visualized by exposure to X-ray films with the ECL system (GE Healthcare UK Ltd., Buckinghamshire, UK).

\section{Immunohistochemical Analyses}

We reviewed the medical records of all consecutive patients who underwent resection with curative intent for pancreatic cancer from 1995 to 2004 at Keio University Hospital, Japan. For immunohistochemistry, 73 invasive ductal pancreatic adenocarcinomas were analyzed. Formalin-fixed and paraffin-embedded sections were incubated with anti-CAP1 antibody overnight at $4^{\circ} \mathrm{C}$. The primary antibody was visualized using an ImmPRESS Anti-Rabbit Ig Kit (Vector Laboratories,
Burlingame, CA, USA) with diaminobenzidine. The sections were counterstained with hematoxylin and then mounted. Histologic diagnoses were made according to the Japanese Pancreas Society classification. ${ }^{25}$

\section{Statistical Analyses}

The $\chi^{2}$-test was used when appropriate to determine the correlations between clinicopathological variables and CAP1 immunolabeling. Survival rates were calculated by the Kaplan-Meier method, and the log-rank test was applied. In the 73 consecutive patients, 4 patients who suffered in-hospital death and 15 patients without follow-up data were excluded for survival analysis. Taken together, follow-up data of 54 patients were utilized for survival analysis. All statistical analyses were performed using the SPSS software (SPSS Inc., Chicago, IL, USA).

\section{Immunocytochemical Analyses}

Human pancreatic cancer cell lines PANC-1, CFPAC-1, and Hs 766T were obtained from the American Type Culture Collection (Manassas, VA, USA). Cells cultured on culture slides (BD Biosciences, San Diego, CA, USA) were fixed with $3.7 \%$ formaldehyde in PBS, permeabilized with $0.1 \%$ Triton $\mathrm{X}-100$ in PBS, and probed with anti-CAP1 antibody overnight at $4^{\circ} \mathrm{C}$. The slides were rinsed with PBS, covered with FITC-labeled secondary antibody with rhodamine-phalloidin (Invitrogen), and visualized using an Axiovert 200 microscope, an AxioCam CCD camera, and the AxioVision software (Carl Zeiss MicroImaging Inc., Tokyo, Japan). Infiltrating lymphocytes served as a positive control for CAP1 staining.

\section{RNA Interference}

For knockdown of CAP1, two siRNA molecules (siCAP1A and siCAP1B) were synthesized by QIAGEN. Their target sequences were AAACCGAGTCCTCAAAGAGTA and AAAC AGATGGCTGCCATGCTT, respectively. As a control, negative control siRNA (nonsilencing siRNA) was purchased from QIAGEN. CFPAC-1 and PANC-1 cells were dispersed into collagen I-coated six-well plates at $5 \times 10^{4}$ cells/well and cultured overnight under normal conditions. The following day, cells were transfected with siRNA in serum-free medium using Oligofectamine (Invitrogen) for $4 \mathrm{~h}$ at $37^{\circ} \mathrm{C}$ with $5 \%$ $\mathrm{CO}_{2}$. After 4-h transfection, an equal volume of medium, supplemented with $20 \%$ fetal bovine serum (FBS), was added and the transfectants were cultured until further analyses. To observe lamellipodium formation, the transfectants $(48 \mathrm{~h}$ after transfection) were serum starved for $24 \mathrm{~h}$, and then stimulated with serum (by changing serum-free medium for medium supplemented with $10 \%$ FBS) for $30 \mathrm{~min}$.

\section{Migration Assay}

CFPAC-1 and PANC-1 cells transfected for $48 \mathrm{~h}$ with siCAP1A, siCAP1B, or negative control siRNA were suspended in RPMI medium (Invitrogen) supplemented with 

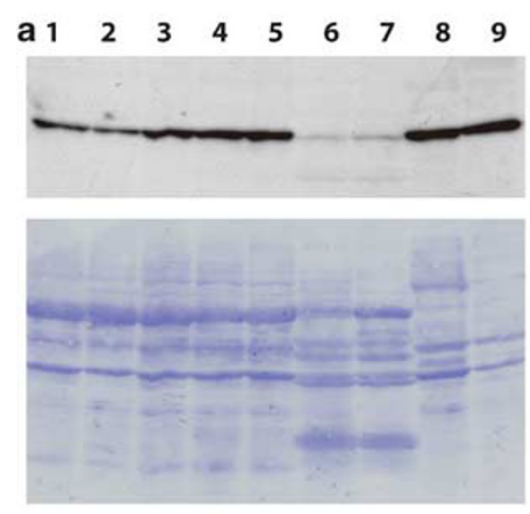
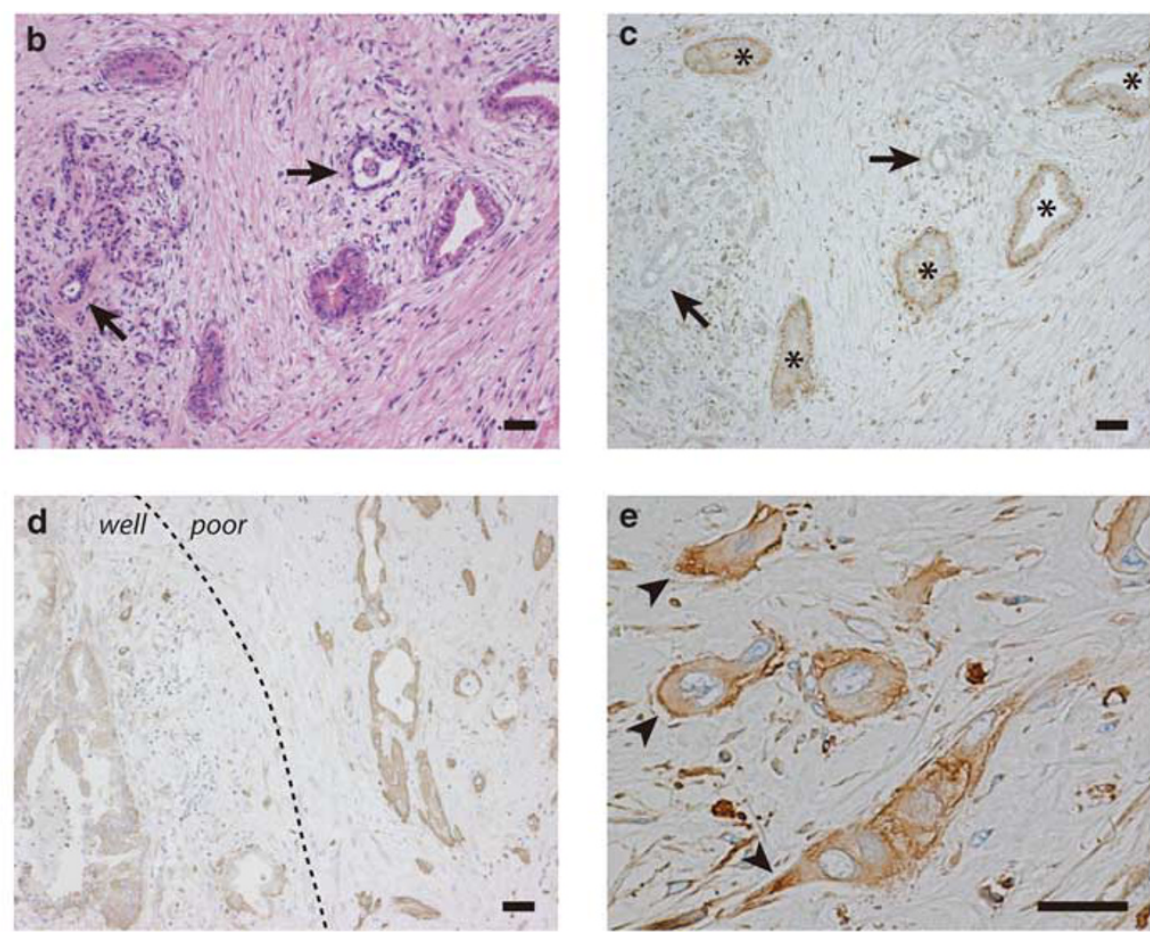

Figure 1 CAP1 overexpression in pancreatic cancers. (a) Expression level of CAP1 protein in pancreatic cancer tissues (lanes 1-5), non-neoplastic pancreas tissues (lanes 6 and 7), and pancreatic cancer cells, Hs 766T (lane 8) and PANC-1 (lane 9) was assessed by Western blot analysis (top). Equal protein load in samples was confirmed by Coomassie brilliant blue staining (bottom). (b) Pancreatic cancer tissue stained with hematoxylin and eosin containing nonneoplastic pancreatic ducts (arrows). (c) Most tumor cells (asterisks) were CAP1-positive whereas non-neoplastic duct cells (arrows) were negative. (d) In a lesion containing well- and poorly differentiated tumor cells, CAP1 was highly expressed in poorly differentiated cells. (e) CAP1 was detected strongly at the edge of tumor cells (arrowheads). Scale bars $=25 \mu \mathrm{m}$.

$0.5 \%$ FBS, and $5 \times 10^{4}(\mathrm{CFPAC}-1)$ or $2 \times 10^{4}(\mathrm{PANC}-1)$ cells treated with each siRNA were dispersed to each of three independent upper chambers of BD BioCoat Control Culture Inserts (six-well plates, pore size $=8 \mu \mathrm{m}$; BD Biosciences). Culture medium containing 10\% FBS was added to the bottom chamber. Following 24-h (CFPAC-1) or 20-h (PANC-1) culture at $37^{\circ} \mathrm{C}$ with $5 \% \mathrm{CO}_{2}$, the cells on the upper surface of the membrane in the chambers were removed by rubbing with cotton-tipped swabs. Cells migrating to the lower surface of the membrane were stained with Diff-Quik (Kokusai Shiyaku, Kobe, Japan). The number of migrated cells on three independent membranes was counted under microscope. Percent migration was represented as the mean number of migrated cells with each siRNA treatment divided by the mean number of migrated control cells.

\section{RESULTS}

\section{Gene-Expression Profiling of Pancreatic Cancer Xenografts}

We compared the gene-expression profiles of 12 pancreatic cancer xenografts with those of normal human pancreas tissues. As the samples were xenografts of human pancreatic cancer tissues grown in SCID mice, tumor cells derived from humans and stromal cells from mice coexisted in each sam- ple. As Affymetrix's GeneChip microarray can distinguish a one-base difference, the probes can identify cRNA derived from human tumor cells in the samples, although some probes detect cRNA derived from both species. We hybridized cRNA derived from each of the xenografts and normal human pancreas tissues to human gene-expression array HG-U133A. More than 10-fold upregulated and highly expressed (signals at $\geq 5000$ ) genes in $\geq 10 / 12$ of the xenografts were extracted from about 22000 probe sets. To improve the value as marker molecules, probe sets with a signal at $>1000$ in normal human pancreas were then filtered out, and finally 57 genes were extracted (Supplementary Table 1). Half of them (26 genes indicated by asterisks in Supplementary Table 1) have been listed as overexpressed genes in pancreatic cancers by microarray analyses in published papers. ${ }^{13-18}$ This agreement suggested that xenografted specimens are able to serve as a panel for pancreatic cancer.

\section{CAP1 Overexpression in Pancreatic Cancers}

As the rapid turnover of actin filament is one of the important factors involved in cell migration and invasion, we focused on a gene encoding an actin-binding protein, CAP1, commonly overexpressed in xenografts. To confirm CAP1 protein expression in pancreatic cancers, Western blot analysis of CAP1 was performed using surgical specimens 
Table 1 Characteristics of 73 pancreatic cancer patients on the basis of CAP1-immunolabeling

\begin{tabular}{|c|c|c|c|}
\hline & \multicolumn{2}{|c|}{ CAP1-positive rate } & \multirow{2}{*}{$\begin{array}{l}P \text { value } \\
\left(\chi^{2} \text {-test }\right.\end{array}$} \\
\hline & $<75 \%$ & $\geq 75 \%$ & \\
\hline \multicolumn{4}{|l|}{ Age (years old) } \\
\hline$<65$ & 11 & 19 & 0.213 \\
\hline$\geq 65$ & 10 & 33 & \\
\hline \multicolumn{4}{|l|}{ Gender } \\
\hline Male & 12 & 34 & 0.509 \\
\hline Female & 9 & 18 & \\
\hline \multicolumn{4}{|l|}{ Differentiation } \\
\hline Well & 5 & 17 & 0.325 \\
\hline Moderately+poorly & 16 & 35 & \\
\hline \multicolumn{4}{|l|}{ Stroma } \\
\hline Intermediate & 4 & 21 & 0.082 \\
\hline Scirrhous & 17 & 31 & \\
\hline \multicolumn{4}{|l|}{ Infiltrative type } \\
\hline$\beta$ & 8 & 19 & 0.901 \\
\hline$\gamma$ & 13 & 33 & \\
\hline \multicolumn{4}{|l|}{ Lymphatic invasion } \\
\hline $0-1$ & 16 & 27 & 0.056 \\
\hline $2-3$ & 5 & 25 & \\
\hline \multicolumn{4}{|l|}{ Vascular invasion } \\
\hline $0-1$ & 12 & 23 & 0.317 \\
\hline $2-3$ & 9 & 29 & \\
\hline \multicolumn{4}{|l|}{ Neural invasion } \\
\hline $0-1$ & 13 & 14 & 0.005 \\
\hline $2-3$ & 8 & 38 & \\
\hline \multicolumn{4}{|l|}{ Resection margin } \\
\hline Negative & 18 & 31 & 0.032 \\
\hline Positive & 3 & 21 & \\
\hline \multicolumn{4}{|c|}{ Pathological tumor status (T) } \\
\hline $1-3$ & 13 & 26 & 0.356 \\
\hline 4 & 8 & 26 & \\
\hline \multicolumn{4}{|c|}{ Pathological node status ( $N$ ) } \\
\hline $0-1$ & 19 & 32 & 0.015 \\
\hline $2-3$ & 2 & 20 & \\
\hline \multicolumn{4}{|c|}{ Pathological metastasis status (M) } \\
\hline 0 & 20 & 44 & 0.211 \\
\hline 1 & 1 & 8 & \\
\hline
\end{tabular}

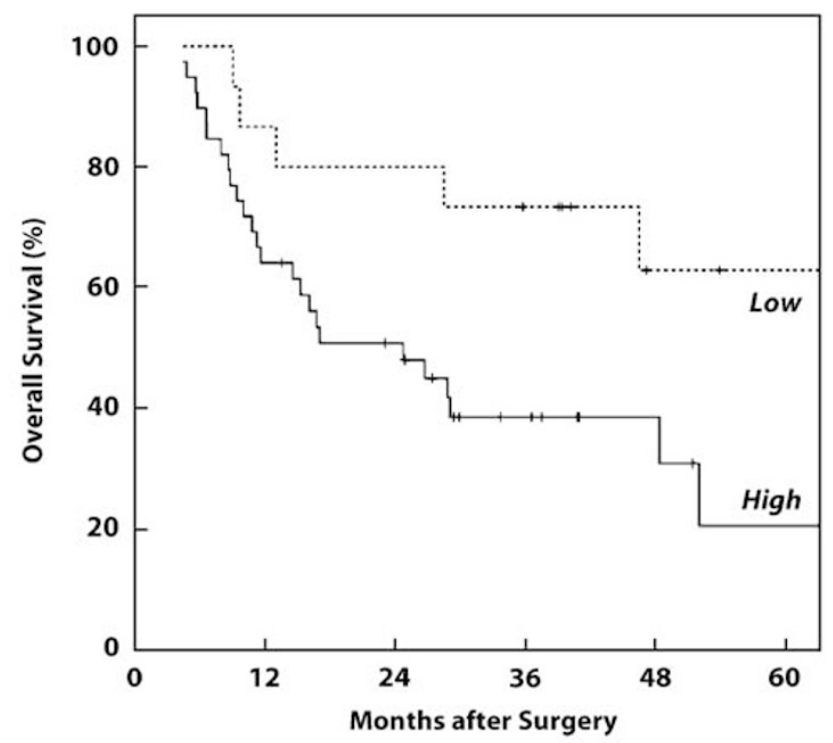

\begin{tabular}{lcccccc}
\multicolumn{1}{l}{ Number at risk } & \multicolumn{1}{c}{ (12 } & 24 & 36 & 48 & 60 \\
\hline Month & 0 & 13 & 13 & 10 & 5 & 4 \\
CAP1 low & 15 & 25 & 18 & 9 & 5 & 2 \\
\hline
\end{tabular}

Figure 2 Kaplan-Meier survival analysis. Overall survival (\%) of patients with $\geq 75 \%$ (high) and $<75 \%$ (low) CAP1-positive rate are indicated by solid and dotted lines, respectively.

(Figure 1a). Pancreatic cancer tissues (lanes 1-5) and cells (Hs 766T and PANC-1 in lanes 8 and 9, respectively) highly expressed CAP1 protein in comparison with non-neoplastic tissues of the pancreas (lanes 6 and 7). Immunohistochemical (IHC) analyses of CAP1 on pancreatic cancer specimens showed that CAP1 was highly expressed in tumor cells (asterisks in Figure 1c) as compared with pancreatic ducts (arrows in Figure 1c) and acini (73/73 cases). On the other hand, some inflammatory cells exhibited strong CAP1 positivity. In some lesions containing both well- and poorly differentiated tumor cells, poorly differentiated cells showed strong staining for CAP1 as compared with adjacent well-differentiated cells (Figure 1d). Furthermore, strong staining for CAP1 was shown, especially at the edge of the cells (arrowheads in Figure 1e).

\section{CAP1 Overexpression Associates with Poor Prognosis}

When CAP1 positivity was defined as stronger staining in tumor cells than in normal ductal cells, the mean rate of CAP1-positive tumor cells was $78.5 \%$ (standard deviation $=17.9$ ) in 73 cases of pancreatic cancer. We classified the 73 cases into two groups, a higher level CAP1-expression group ( $\geq 75 \%$ CAP1-positive rate) and a lower level CAP1-expression group $(<75 \%)$. As shown in Table 1, the CAP1-positive rate was significantly associated 

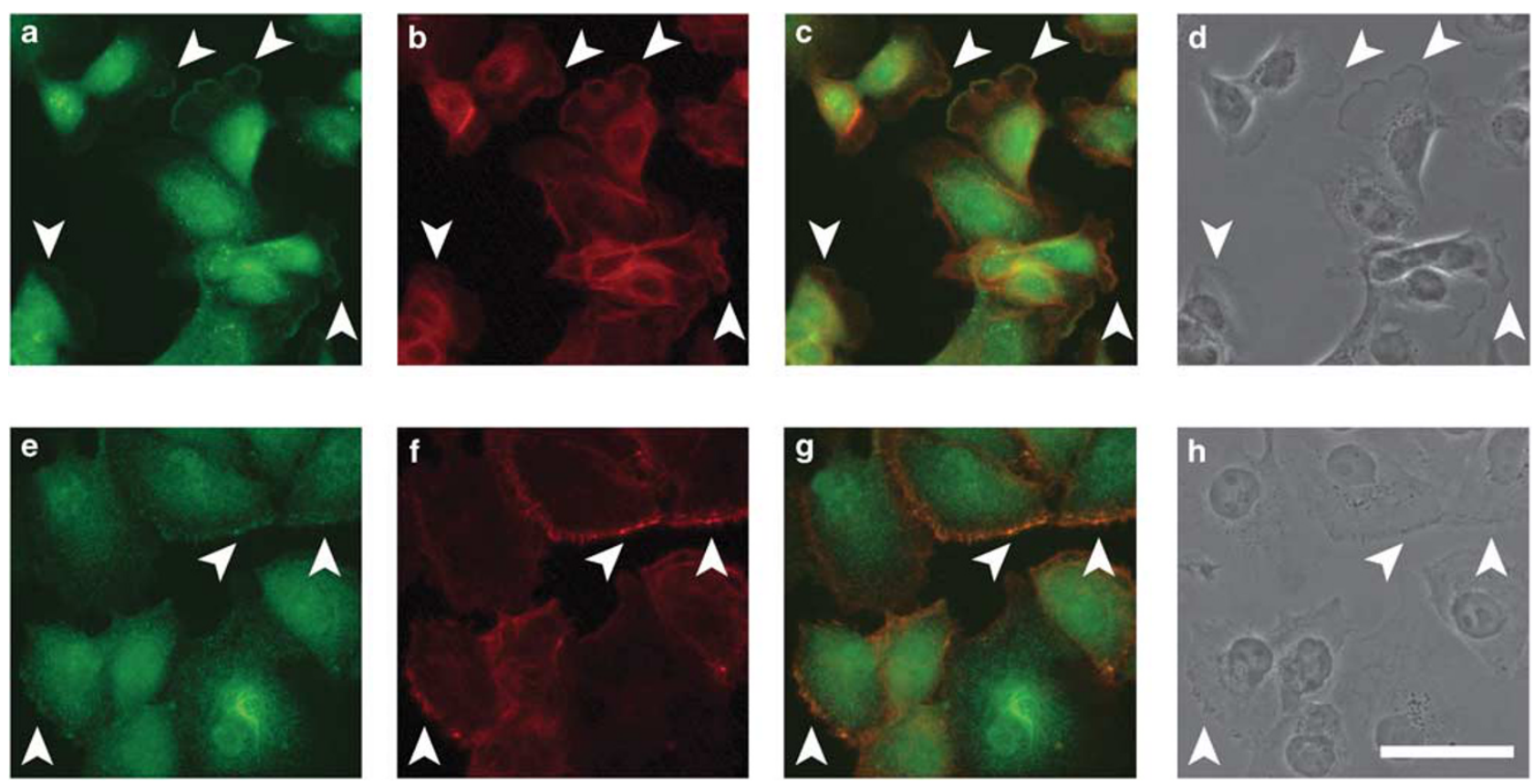

Figure 3 Subcellular localization of CAP1. CFPAC-1 (a-d) and PANC-1 (e-h) cells probed with anti-CAP1 antibody (a, e) and phalloidin-rhodamine (b, f). (c, g) Merged images. (d, h) Phase-contrast images. Arrowheads indicate the leading edge of the lamellipodium with CAP1-actin colocalization. Scale bar $=50 \mu \mathrm{m}$.

with neural invasion $\left(P=0.005\right.$ by $\chi^{2}$-test $)$, resection margin $(P=0.032)$, and lymph node metastasis $(P=0.015)$. KaplanMeier survival analysis revealed the poor outcome of patients in the high-level CAP1-expression group (Figure 2), and 5 -year survival rates were $21 \%$ in the high level group and $63 \%$ in the low level group $(P=0.021, \log$-rank test $)$. These results indicate that the expression level of CAP1 is associated with invasion to neurons and lymph nodes, which can be associated with shorter survival of patients with pancreatic cancer.

\section{Defect in Lamellipodium Formation by CAP1 Knockdown}

By immunocytochemical analyses (Figure 3), CAP1 was detected mainly in the perinuclear cytoplasm. In cells spreading lamellipodia, CAP1 and actin were colocalized to the leading edge of the lamellipodium (arrowheads). As lamellipodium formation is seen in migrating cells, CAP1 localization to the leading edge of the lamellipodium seems to be involved in cell migration.

To analyze the role of CAP1 in cell function, knockdown of CAP1 in pancreatic cancer cells was performed using two small interfering RNA (siRNA) molecules (siCAP1A and siCAP1B), because CAP1 was highly expressed in all eight pancreatic cancer-derived cells examined by microarray analyses (microarray data are accessible from our in-house database, GeMDBJ). Figure 4a shows the results of Western blot in which CAP1 expression in CFPAC-1 and PANC-1 transfectants was reduced with either siRNA molecule. In the two siRNA molecules, siCAP1A seems to be more effective in suppressing CAP1 protein than siCAP1B. Considering the localization of CAP1 to the leading edge of the lamellipodium, to examine the role of CAP1 in lamellipodium formation, knockdown cells were stimulated with serum. Culture medium was exchanged to RPMI 1640 medium without serum 2 days after siRNA transfection. After serum starvation for $24 \mathrm{~h}$, the transfectants were stimulated with serum by changing the medium to normal culture medium supplemented with 10\% FBS. By serum withdrawal, lamellipodium formation was suppressed in CFPAC-1 cells transfected with any siRNA (Figure 4b). Within 15 min after serum stimulation, control cells extended lamellipodia around the circumference of the cell clusters (arrowheads), whereas lamellipodium protrusion of CAP1-knockdown cells was still suppressed or was only modest (arrows). In PANC-1 cells, shrunken lamellipodia by serum withdrawal began to extend by serum addition, although the recovery of lamellipodium extension was delayed in CAP1-knockdown cells compared with control cells (Supplementary Figure 1). These results demonstrate that CAP1 knockdown affected lamellipodium formation.

\section{Reduction of Cell Motility by CAP1 Knockdown}

As protruding lamellipodia are seen in migrating cells, the role of CAP1 in cell motility was evaluated by migration assays using CAP1-knockdown cells (Figure 4c). In both cell lines (CFPAC-1 and PANC-1), the migrated cell number was decreased by CAP1 knockdown. Of note, the ratio of the 
a
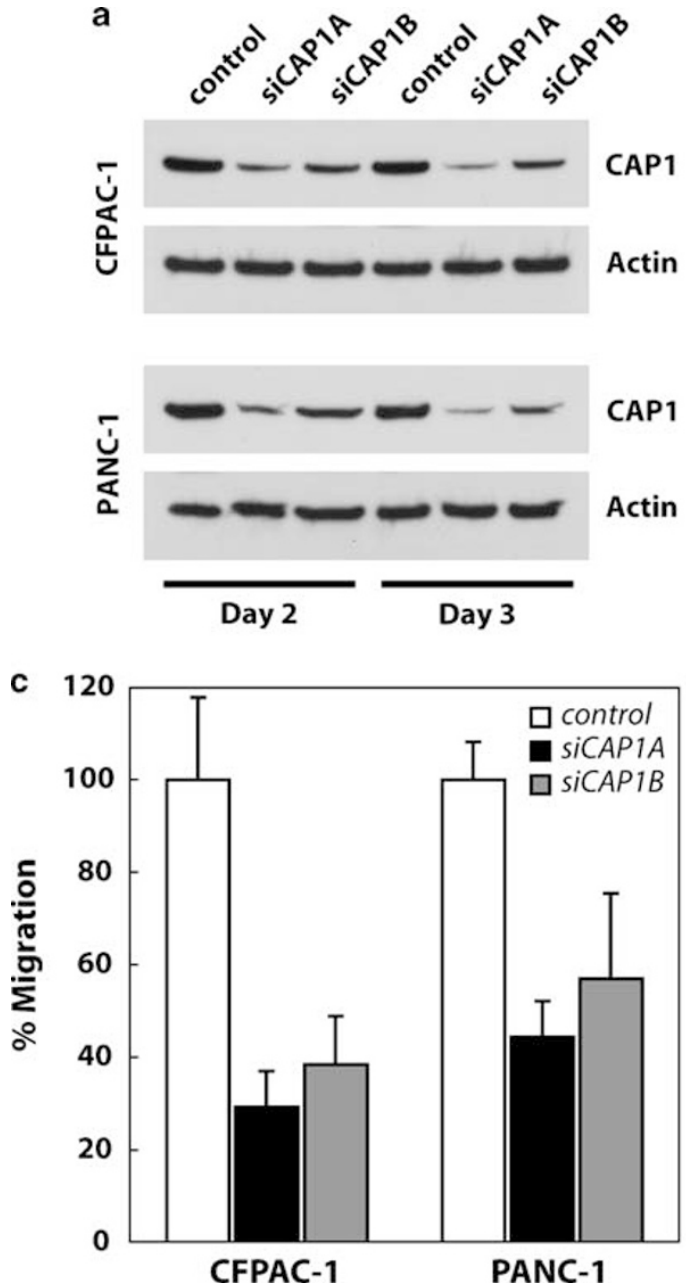

b
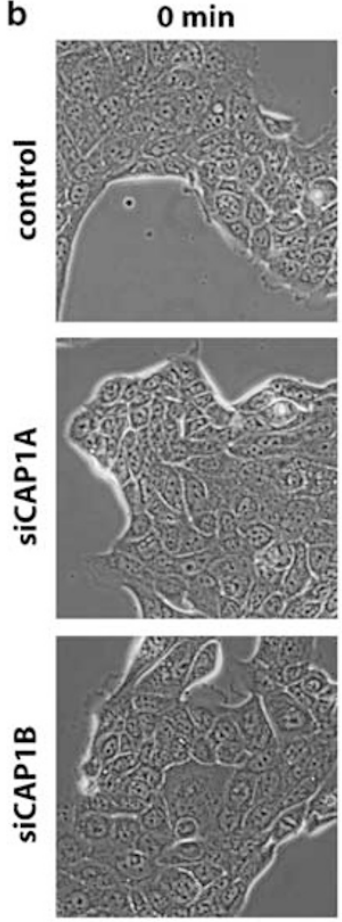
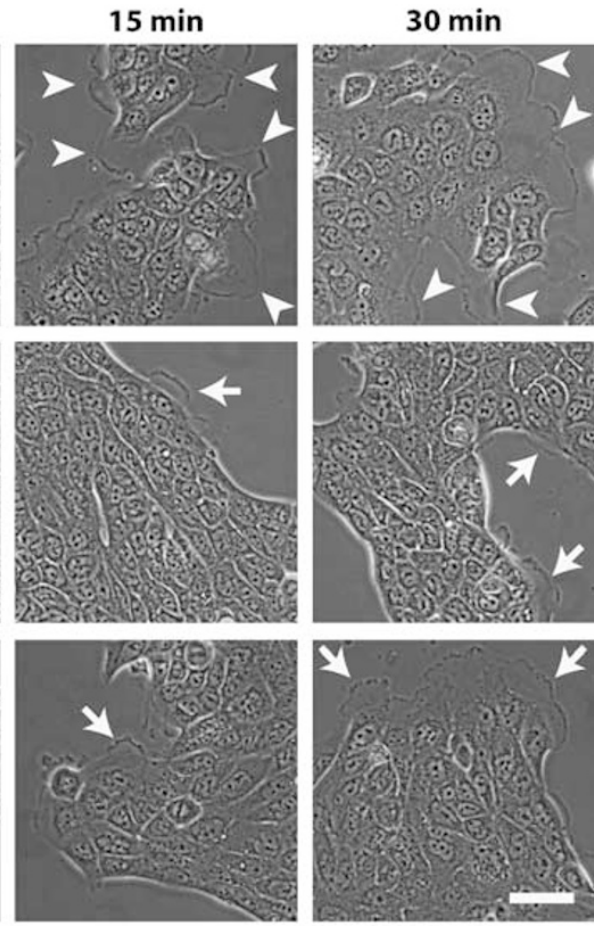

Figure 4 Effects of CAP1 knockdown. (a) Western blot analyses of CAP1 and actin using lysates of CFPAC-1 cells and of PANC-1 cells treated for 2 and 3 days with siCAP1A, siCAP1B, or negative control siRNA. (b) Microscopy of CFPAC-1 transfectants serum-starved (0 min) and serum-stimulated for 15 and 30 min. Scale bar $=50 \mu \mathrm{m}$. Arrowheads and arrows indicate lamellipodia of control cells and CAP1-knockdown cells, respectively. (c) Migration assays showed reduced motility of CFPAC-1 and PANC-1 cells transfected with siCAP1A and siCAP1B as compared with control cells. Percent migration is represented as the mean number of migrated cells per field under a microscope against control cells. Bars reveal standard deviations.

migrated cell number was more decreased in siCAP1A transfectants in comparison with siCAP1B transfectants. This knockdown effect was consistent with that in suppressing CAP1 protein with the two siRNA molecules (Figure 4a). Considering that cell proliferation was not affected by RNAi (Supplementary Figure 2), the decreased number of migrated cells seems to be dependent on the reduced cell motility by CAP1 knockdown. Reduced cell motility by CAP1 knockdown was seen in another pancreatic cancer cell line, AsPC-1 (data not shown).

\section{DISCUSSION}

As a result of microarray analyses of pancreatic cancer xenografts, CAP1 was extracted as a candidate marker for pancreatic cancer. And IHC analyses showed its overexpression in $73 / 73$ cases of pancreatic cancer examined. These results demonstrate that CAP1 overexpression was a common feature in pancreatic cancers, and suggest that xenografting is likely to be a useful method for profiling cancers containing abundant stromal cells. IHC analyses also showed heterogeneous expression of CAP1, which was upregulated in poorly differentiated tumor cells than in welldifferentiated tumor cells. It is reported that reduced E-cadherin expression is detected frequently in poorly differentiated tumor cells than in well-differentiated tumor cells, ${ }^{26,27}$ and reduced E-cadherin expression is regarded as a predictor of poor outcome in pancreatic cancer. It is possible that a reciprocal transcriptional regulation of CAP1 and E-cadherin occurs in poorly differentiated tumor cells. Expression levels of CAP1 in pancreatic cancer specimens were significantly correlated with the presence of neural invasion and lymph node metastasis. Neural invasion and lymph node metastasis of pancreatic cancer are associated with poor outcome, ${ }^{5-7}$ and patients with high-level CAP1 expression had a poor outcome. 
These results suggest that CAP1 overexpression promoted invasion and metastasis, which can be associated with the poor outcome of patients with pancreatic cancer.

Adenylate cyclase-associated proteins are multifunctional proteins with several structural domains involved in actin binding, adenylate cyclase association, $\mathrm{SH} 3$ binding, and oligomerization. ${ }^{20}$ Yeast CAP is involved in adenylate cyclase activation in the RAS signaling pathway; ${ }^{28}$ however, its function is not apparent in mammals. Further studies are needed to elucidate the molecular function of CAP1 in the RAS signaling. Both mammal and yeast CAPs interact with actin $^{29}$ and play a role in actin turnover. ${ }^{23}$ In some reports about CAP1 mutations, the predicted functions of CAP1 have been identified from their phenotypes. Deletion of yeast CAP results in an abnormally large cell size, random budding pattern, and abnormal actin distribution. ${ }^{30,31}$ A CAP knockout mutant of Dictyostelium revealed aberrations in cell polarization, F-actin organization, and phototaxis. ${ }^{32}$ Loss of Drosophila CAP results in various developmental defects and problems in maintaining oocyte polarity. ${ }^{33,34}$ In addition to those genetic studies, CAP1 knockdown results in aberrant cell morphology and reduced migration in mouse cells. ${ }^{35}$ These reports suggest that a failure of actin turnover by aberrations in CAP1 orthologs induces abnormalities in cell morphology, polarity, and motility. In our study, protruding of the lamellipodium was induced by serum stimulation and was inhibited by CAP1 knockdown, suggesting that CAP1 was involved in serum-induced lamellipodium formation. In addition, cell motility was reduced by CAP1 knockdown. As remodeling of the actin filament and formation of the actin meshwork occurred at the leading edge of the lamellipodium where CAP1 and actin colocalized, retardation of actin turnover by CAP1 knockdown seems to be a cause of the defect in lamellipodium formation and reduced cell motility. Considering that expression levels of CAP1 in clinical specimens were associated with neural invasion and lymph node metastasis, it seems that CAP1 overexpression was involved in enhanced cell motility and promoted invasion and metastasis of pancreatic cancer.

In conclusion, CAP1 was shown to be commonly overexpressed in pancreatic cancers, and to be associated with enhanced cell motility; therefore, CAP1 overexpression may be involved in the aggressive behavior of pancreatic cancers.

Supplementary Information accompanies the paper on the Laboratory Investigation website (http://www.laboratoryinvestigation.org)

\section{ACKNOWLEDGEMENTS}

We thank Dr Moriyama for the kind gift of polyclonal antibody against human CAP1, and Rie Itoh, Kaoru Onozato, and Hiroshi Suzuki for their technical assistance.

Grant support: The Program for the Promotion of Fundamental Studies in Health Sciences of the Pharmaceuticals and Medical Devices Agency (PMDA); Grant-in-aid from the 21st Century Center of Excellence program and Scientific Research on Priority Areas from the Ministry of Education, Culture, Sports, Science and Technology of Japan; Grant-in-aid from the Third Term Comprehensive 10-Year Strategy for Cancer Control from the
Ministry of Health, Labor and Welfare of Japan; Grant-in-aid for Cancer Research from the Foundation for Promotion of Cancer Research.

\section{DISCLOSURE/CONFLICT OF INTEREST}

The authors declared no conflict of interests.

1. James TA, Gibbs JF. Pancreatic cancer screening: identifying premalignant disease. Future Oncol 2005;1:191-195.

2. Matsuda T, Marugame $\mathrm{T}$, Kamo Kl, et al. Cancer incidence and incidence rates in Japan in 2002: based on data from 11 populationbased cancer registries. Jpn J Clin Oncol 2008;38:641-648.

3. Warshaw A, Fernandez-del Castillo C. Pancreatic carcinoma. N Engl J Med 1992;326:455-465.

4. Conlon KC, Klimstra DS, Brennan MF. Long-term survival after curative resection for pancreatic ductal adenocarcinoma. Clinicopathologic analysis of 5-year survivors. Ann Surg 1996;223:273-279.

5. Nakao A, Harada A, Nonami $T$, et al. Lymph node metastasis in carcinoma of the body and tail of the pancreas. Br J Surg 1997;84:1090-1092.

6. Sperti C, Pasquali C, Piccoli $A$, et al. Survival after resection for ductal adenocarcinoma of the pancreas. Br J Surg 1996;83:625-631.

7. Tsiotos GG, Farnell MB, Sarr MG. Are the results of pancreatectomy for pancreatic cancer improving. World J Surg 1999;23:913-919.

8. Scarpa A, Capelli P, Villaneuva A, et al. Pancreatic cancer in Europe: Ki-ras gene mutation pattern shows geographical differences. Int J Cancer 1994;57:167-171.

9. Moore PS, Sipos B, Orlandini S, et al. Genetic profile of 22 pancreatic carcinoma cell lines. Analysis of K-ras, p53, p16 and DPC4/Smad4. Virchows Arch 2001;439:798-802.

10. Caldas C, Hahn SA, da Costa LT, et al. Frequent somatic mutations and homozygous deletions of the p16 (MTS1) gene in pancreatic adenocarcinoma. Nat Genet 1994;8:27-32.

11. Ruggeri B, Zhang SY, Caamano J, et al. Human pancreatic carcinomas and cell lines reveal frequent and multiple alterations in the p53 and $\mathrm{Rb}-1$ tumor-suppressor genes. Oncogene 1992;7:1503-1511.

12. Hahn SA, Schutte M, Hoque AT, et al. DPC4, a candidate tumor suppressor gene at human chromosome 18q21.1. Science 1996;271:350-353.

13. Crnogorac-Jurcevic T, Efthimiou E, Nielsen T, et al. Expression profiling of microdissected pancreatic adenocarcinomas. Oncogene 2002;21:4587-4594.

14. lacobuzio-Donahue CA, Maitra A, Shen-Ong GL, et al. Discovery of novel tumor markers of pancreatic cancer using global gene expression technology. Am J Pathol 2002;160:1239-1249.

15. lacobuzio-Donahue CA, Ashfaq R, Maitra A, et al. Highly expressed genes in pancreatic ductal adenocarcinomas: a comprehensive characterization and comparison of the transcription profiles obtained from three major technologies. Cancer Res 2003;63:8614-8622.

16. Logsdon $C D$, Simeone DM, Binkley $C$, et al. Molecular profiling of pancreatic adenocarcinoma and chronic pancreatitis identifies multiple genes differentially regulated in pancreatic cancer. Cancer Res 2003;63:2649-2657.

17. Tan ZJ, Hu XG, Cao GS, et al. Analysis of gene expression profile of pancreatic carcinoma using CDNA microarray. World J Gastroenterol 2003;9:818-823.

18. Grutzmann R, Foerder M, Alldinger I, et al. Gene expression profiles of microdissected pancreatic ductal adenocarcinoma. Virchows Arch 2003;443:508-517.

19. Loukopoulos $P$, Kanetaka K, Takamura M, et al. Orthotopic transplantation models of pancreatic adenocarcinoma derived from cell lines and primary tumors and displaying varying metastatic activity. Pancreas 2004;29:193-203.

20. Hubberstey AV, Mottillo EP. Cyclase-associated proteins: CAPacity for linking signal transduction and actin polymerization. FASEB J 2002;16:487-499.

21. Loisel TP, Boujemaa R, Pantaloni D, et al. Reconstitution of actin-based motility of Listeria and Shigella using pure proteins. Nature 1999;401:613-616.

22. Pollard TD, Blanchoin L, Mullins RD. Molecular mechanisms controlling actin filament dynamics in nonmuscle cells. Annu Rev Biophys Biomol Struct 2000;29:545-576. 
23. Moriyama K, Yahara I. Human CAP1 is a key factor in the recycling of cofilin and actin for rapid actin turnover. J Cell Sci 2002;115: 1591-1601.

24. Shibata R, Mori T, Du W, et al. Overexpression of cyclase-associated protein 2 in multistage hepatocarcinogenesis. Clin Cancer Res 2006; 12:5363-5368.

25. Japanese Pancreas Society. Classification of Pancreatic Carcinoma, 2nd English edn. Kanehara and Co. Ltd: Tokyo, 2003.

26. Pignatelli M, Ansari TW, Gunter P, et al. Loss of membranous E-cadherin expression in pancreatic cancer: correlation with lymph node metastasis, high grade, and advanced stage. J Pathol 1994;174: 243-248.

27. Kuniyasu H, Ellis LM, Evans DB, et al. Relative expression of E-cadherin and type IV collagenase genes predicts disease outcome in patients with resectable pancreatic carcinoma. Clin Cancer Res 1999;5: 25-33.

28. Field J, Vojtek A, Ballester R, et al. Cloning and characterization of CAP, the $\mathrm{S}$. cerevisiae gene encoding the $70 \mathrm{kd}$ adenylyl cyclase-associated protein. Cell 1990;61:319-327.
29. Freeman NL, Chen Z, Horenstein J, et al. An actin monomer binding activity localizes to the carboxyl-terminal half of the Saccharomyces cerevisiae cyclase-associated protein. J Biol Chem 1995;270:5680-5685.

30. Gerst JE, Ferguson K, Vojtek A, et al. CAP is a bifunctional component of the Saccharomyces cerevisiae adenylyl cyclase complex. Mol Cell Biol 1991;11:1248-1257.

31. Vojtek A, Haarer B, Field J, et al. Evidence for a functional link between profilin and CAP in the yeast $S$. cerevisiae. Cell 1991;66:497-505.

32. Noegel AA, Blau-Wasser $R$, Sultana $H$, et al. The cyclase-associated protein CAP as regulator of cell polarity and CAMP signaling in Dictyostelium. Mol Biol Cell 2004;15:934-945.

33. Baum B, Li W, Perrimon N. A cyclase-associated protein regulates actin and cell polarity during Drosophila oogenesis and in yeast. Curr Biol 2000;10:964-973.

34. Baum B, Perrimon N. Spatial control of the actin cytoskeleton in Drosophila epithelial cells. Nat Cell Biol 2001;3:883-890.

35. Bertling $E$, Hotulainen $P$, Mattila PK, et al. Cyclase-associated protein 1 (CAP1) promotes cofilin-induced actin dynamics in mammalian nonmuscle cells. Mol Biol Cell 2004;15:2324-2334. 